\title{
Besser kein Insulin für Menschen mit Typ-2-Diabetes?
}

\author{
Bei Patienten mit Typ-2-Diabetes sollte eine Insulintherapie so lange wie möglich hinausgezö- \\ gert und, falls unvermeidlich, so niedrig dosiert wie möglich und grundsätzlich mit Metformin \\ kombiniert werden. Das fordert jedenfalls Professor Craig Currie, Pharmakoepidemiologe an der \\ Universität Cardiff.
}

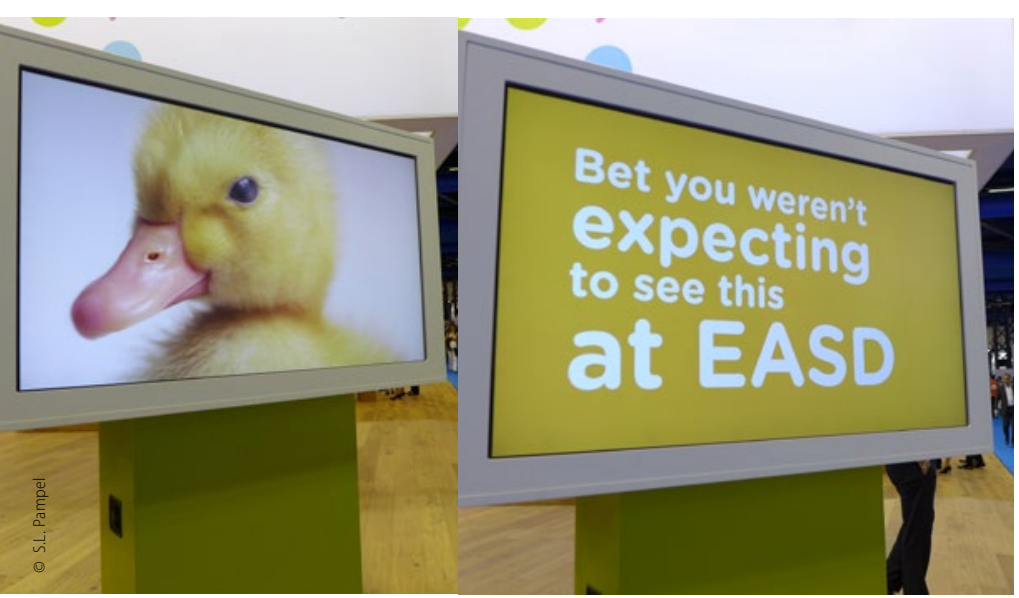

Niedliche Tierchen in einer Kongressausstellung und unkonventionelle Meinungen - beides nicht unbedingt zu erwarten.

Die gute Nachricht lautet: Die Lebenserwartung von Typ-2-Diabetikern ist in den letzten Jahren kontinuierlich gestiegen und beträgt heute fast zehn Jahre mehr als zu Beginn der 1990erJahre. An der Qualität der antihyperglykämischen Therapie kann das kaum liegen, wie Currie meint. Denn bei der Blutzuckerkontrolle gab es wenig Fortschritt, ganz egal welche therapeutische Strategie verfolgt wurde. Der durchschnittliche $\mathrm{HbA}_{1 \mathrm{c}}$-Wert liegt etwa einen halben Prozentpunkt niedriger als vor 25 Jahren.

Der $\mathrm{HbA}_{1 \mathrm{c}}$ interessiere Patienten ohnehin nur am Rande, so der Pharmakoepidemiologe: „Solche intermediären Parameter sind in meinen Augen passé, auch als Endpunkte für klinische Studien." Entscheidend und patientenrelevant seien kardiovaskuläre Komplikationen, weil sie das Leben verkürzen und die Lebensqualität der Überlebenden beeinträchtigen. Sechs von zehn Typ-Diabetikern sterben an makrovaskulären Komplikationen - daran hat sich seit Jahren wenig geändert. In der Konsequenz verlangen die Arzneimittelbehörden FDA und EMA zumindest den Nachweis der kardiovaskulären Sicherheit für die Zulassung neuer Antidiabetika.

\section{Hypoglykämierisiko entscheidend}

Welchen Beitrag die blutzuckersenkende Therapie zur kardiovaskulären Risikominimierung leisten kann - neben anderen pharmakologischen Interventionen wie Plättchenhemmung, Blutdruck- und Lipidsenkung -, ist nach wie vor nicht sicher geklärt. Currie ist jedoch der festen Überzeugung, dass sich die Antidiabetika hier unterscheiden und dass das Hypoglykämierisiko unter den verschiedenen Wirkstoffklassen eine entscheidende Rolle spielt. Er erinnerte daran, dass Insulin ein so hohes
Hypoglykämierisiko birgt wie kein anderes Antidiabetikum es liegt bei etwa 12 pro 100 Patientenjahre, während Sulfonylharnstoffe auf knapp 1 pro 100 Patientenjahre kommen und andere Wirkstoffklassen nur selten Hypos induzieren. In den großen Endpunktstudien ACCORD, ADVANCE und VADT waren in den intensiv therapierten Armen mehr Hypoglykämien beobachtet worden, und die Inzidenz war umso höher, je mehr Patienten Insulin erhalten hatten, berichtete Currie.

\section{Besser kein $\mathrm{HbA}_{1 c}$-Wert unter 7\%?}

Post-hoc-Analysen ergaben für alle drei Studien einen klaren Zusammenhang zwischen der Inzidenz schwerer Hypoglykämien und Gesamt- wie kardiovaskulärer Mortalität. In VADT erwiesen sich Hypos sogar als stärkerer Prädiktor für kardiovaskulären Tod (Hazard Ratio 4,0) als vorangegangene vaskuläre Komplikationen (Hazard Ratio 3,1). „Hypoglykämien sollten unter allen Umständen vermieden werden“, forderte Currie.

Currie stellt auch das Konzept infrage, durch intensive Glukosekontrolle die kardiovaskuläre Prognose und die Gesamtsterblichkeit verbessern zu wollen. Seinen eigenen Observationsstudien zufolge existiert auch beim $\mathrm{HbA}_{1 \mathrm{c}}$ eine J-Kurve, wonach der optimale $\mathrm{HbA}_{1 \mathrm{c}}$-Bereich sowohl für Patienten mit oralen Antidiabetika als auch mit Insulin um zwischen 7,0 und 8,5\% liegt. „Andere große populationsbezogene Studien bestätigen unsere Daten“, betonte Currie. In diesem Punkt erntete er jedoch Kritik aus dem Auditorium: Er habe eine ganze Reihe von Studien unterschlagen, die das Gegenteil zeigten, nämlich dass Gefäßkomplikationen und Sterbefälle bei strikter Stoffwechseleinstellung sehr wohl zurückgehen. Currie beharrte jedoch darauf, dass eine $\mathrm{HbA}_{1 \mathrm{c}}$-Senklung auf $<7 \%$ nur sehr vorsichtig erfolgen sollte.

Außerdem sollte seiner Ansicht nach möglichst ganz auf eine Insulinisierung verzichtet werden. Einer weiteren Beobachtungsstudie zufolge schnitten orale Antidiabetika immer dann hinsichtlich der Gesamtmortalität schlechter ab, wenn sie mit Insulin kombiniert wurden [1]. Das Sterberisiko stieg zudem mit der Insulindosis. Auch hier erntete Currie aber Widerspruch aus dem Kollegenkreis: Er könne schließlich nicht ausschließen, dass die Insulinisierung schlicht ein Zeichen für eine fortgeschrittene Diabeteserkrankung mit mehr Begleit- und Folgeerkrankungen sei.

Manuela Arand

\author{
Literatur: \\ 1. J Clin Endocrinol Metab. 2013 Feb;98(2):668-77.
}

Quelle: EASD/ESC-Symposium: „Lessons learned from glucose lowering trials?" bei der EASD-Jahrestagung, in Stockholm, 14.-18.9.2015 